Article

\title{
Effects of Tung Oil-Based Polyols on the Thermal Stability, Flame Retardancy, and Mechanical Properties of Rigid Polyurethane Foam
}

\author{
Wei Zhou ${ }^{1,2}$, Caiying Bo ${ }^{1,3}$, Puyou Jia ${ }^{1,3}$, Yonghong Zhou ${ }^{1,3,4}$ and Meng Zhang 1,3,4,* \\ 1 Institute of Chemical Industry of Forestry Products, CAF, 16 Suojin North Road, Nanjing 210042, China; \\ vvzhou90@163.com (W.Z.); newstar2002@163.com (C.B.); jiapuyou@163.com (P.J.); \\ yhzhou777@163.com (Y.Z.) \\ 2 Key Lab of Forest Chemical Engineering, SFA, 16 Suojin North Road, Nanjing 210042, China \\ 3 Key Lab of Biomass Energy and Material, Jiangsu Province, 16 Suojin North Road, Nanjing 210042, China \\ 4 Co-Innovation Center of Efficient Processing and Utilization of Forest Resources, Nanjing Forestry \\ University, 159 Longpan Road, Nanjing 210037, China \\ * Correspondence: zhangmeng@icifp.cn; Tel.: +86-025-8548-2520
}

Received: 20 November 2018; Accepted: 24 December 2018; Published: 30 December 2018

\begin{abstract}
A phosphorus-containing tung oil-based polyol (PTOP) and a silicon-containing tung oil-based polyol (PTOSi) were each efficiently prepared by attaching 9,10-dihydro-9-oxa-10-phosphaphenanthrene (DOPO) and dihydroxydiphenylsilane (DPSD) directly, respectively, to the epoxidized monoglyceride of tung oil (EGTO) through a ring-opening reaction. The two new polyols were used in the formation of rigid polyurethane foam (RPUF), which displayed great thermal stability and excellent flame retardancy performance. The limiting oxygen index (LOI) value of RPUF containing $80 \mathrm{wt} \%$ PTOP and $80 \mathrm{wt} \%$ PTOSi was $24.0 \%$ and $23.4 \%$, respectively. Fourier transfer infrared (FTIR), Nuclear Magnetic Resonance (NMR) and thermogravimetric (TG) analysis revealed that DOPO and DPSD are linked to EGTO by a covalent bond. Interestingly, PTOP and PTOSi had opposite effects on $T_{\mathrm{g}}$ and the compressive strength of RPUF, where, with the appropriate loading, the compressive strengths were $0.82 \mathrm{MPa}$ and $0.25 \mathrm{MPa}$, respectively. At a higher loading of PTOP and PTOSi, the thermal conductivity of RPUF increased while the RPUF density decreased. The scanning electron microscope (SEM) micrographs showed that the size and closed areas of the RPUF cells were regular. SEM micrographs of the char after combustion showed that the char layer was compact and dense. The enhanced flame retardancy of RPUF resulted from the barrier effect of the char layer, which was covered with incombustible substance.
\end{abstract}

Keywords: tung oil; DOPO; dihydroxydiphenylsiane; flame retardant; rigid polyurethane foam

\section{Introduction}

The depletion of petroleum reserves is driving the development of polymers and polymer additives from sustainable bio-materials. Polymer materials made from renewable sources have attracted attention around the world [1]. Vegetable oils are particularly promising renewable chemicals for the preparation of polyols and rigid polyurethane foam (RPUF) due to their renewability, universal availability, and environmental compatibility. Vegetable oils have double bonds, ester groups and other reactive sites that can be modified to form new polyol structures [2]. Vegetable oils such as castor oil, palm kernel oil [3,4], rapeseed oil [5], and tung oil [6] have been used to produce RPUF. RPUF has been widely used in materials for building insulation due to its excellent mechanical performance. However, a limitation to the use of RPUF is flammability, which is related to its cellular structure and low density [7,8].

In recent years, much effort has been devoted to increasing the flame-retardant properties of RPUF. Conventional methods of flame-retardant treatment for RPUF include the incorporation of flame-retardant 
additives based on phosphorus, silicone, nitrogen, and halogen compounds. While these flame-retardant additives impart excellent flame retardancy to RPUF, the introduction of larger amounts of flame retardants may have an adverse effect on the mechanical and physical characteristics of RPUF [9]. Furthermore, flame retardant additives may easily mobilize out of the RPUF matrix into the exterior [10]. When burning, flame retardants that contain halogens release a lot of harmful gases and bring about environmental pollution. More importantly, organohalogen compounds fragmenting from the polymers are stable and able to persist and bioaccumulate in the environment, and thus may pose a risk to human health [11]. Consequently, an environmentally friendly reactive flame retardant is needed. The main benefit of reactive flame retardants compared to other flame-retardant additives is that the flame-retardant molecule is linked to the RPUF structure with a covalent bond [12].

The compound 9,10-dihydro-9-oxa-10-phosphaphenanthrene (DOPO) [13] is an environmentally friendly reactive flame retardant that has attracted considerable attention because of its outstanding thermal stability and ability to resist oxidation and hydrolysis [14]. Due to its aromatic and phenanthrene ring structure, DOPO is more thermally and chemically stable than standard organic phosphate, and little toxic gas is released during its combustion [15]. The flame-retardant ability of DOPO has been attributed to the formation of a reactive substance such as $\mathrm{PO}^{-}$, which is released to the gas phase where it engages with $\mathrm{H}$. and $\mathrm{OH}$. groups and disrupts the combustion process [16]. DOPO exhibits prominent gas-phase flame retardant properties during RPUF combustion [17,18]. Modification of RPUF through the incorporation of silicon-containing flame retardants is also recognized as an environmentally friendly method that reduces flammability [19]. Silicone materials show good flame-retardant properties through the formation of char barriers $(\mathrm{Si}-\mathrm{C}, \mathrm{Si}-\mathrm{O})$ at the surface of the polymer at high temperatures [20]. There are two ways to enhance the flame retardancy of RPUF, one of which is the addition of the inorganic silicon such as nano-silica and whisker silicon oxide [21], and the other of which is the introduction of silicon into the structure of polyols using 3-aminopropylmethyldiethoxysilane (APTES), $N$-( $\beta$-aminoethyl)- $\gamma$-aminopropylmethyl dimethoxysilane (KH-602), and $\mathrm{SiO}_{2}$ [22-24]. This has resulted in the development of novel reactive flame retardants for RPUF. Tung oil-based flame-retardant polyols have not been reported previously.

The goal of this work was to develop an innovative RPUF based on the incorporation of a phosphorus-containing tung oil-based polyol (PTOP) and a silicon-containing tung oil-based polyol (PTOSi). The molecular structure of the compounds and the thermal properties of the two new tung oil-based flame-retardant polyols were characterized using FTIR, ${ }^{1} \mathrm{H} N M R,{ }^{31} \mathrm{P}$ NMR, and TG. RPUFs were characterized for their thermal and flame-retardant behaviors using several analytical techniques.

\section{Materials and Methods}

\subsection{Materials}

Tung oil (TO) was provided by Jiangsu Qianglin Bioenergy Material Co., Ltd (Qianglin, China). Glycerol, sodium methoxide and ethyl acetate were obtained from Nanjing Chemical Reagent Co., Ltd (Nanjing, China). Acetic acid was purchased from Nanjing Chemical Reagent Co., Ltd (Nanjing, China). Hydrogen peroxide (30 wt \%) was purchased from Shanghai Linfeng Chemical Reagent Co., Ltd (Shanghai, China). Other materials such as P-toluenesulfonic acid monohydrate (TsOH· $\mathrm{H}_{2} \mathrm{O}$ ), 9,10-dihydro-9-oxa-10-phosphaphenanthrene (DOPO), dihydroxydiphenylsilane (DPSD) and dibutyltin dilaurate (DBTDL) were provided by Aladdin Industrial Corporation (Shanghai, China). Polyether polyol (PPG4110) was purchased from Jiangsu Qianglin Bioenergy Material Co., Ltd (Jiangsu, China); and its hydroxyl value was $403 \mathrm{mg} \mathrm{KOH} / \mathrm{g}$ and its viscosity was $3.6 \mathrm{~Pa} \cdot \mathrm{s}$ at $25^{\circ} \mathrm{C}$. Polyaryl polyisocyanate (PAPI) was provided by Yantai Wanhua Polyurethane Co., Ltd (Yantai, China) with $30.3 \mathrm{wt} \%$ NCO. Foam stabilizer (AK8804) was purchased from Jiangsu Maysta Chemical Co., Ltd (Jiangsu, China). Foaming agent (HFC-365mfc) was obtained from Dongguan Changze Chemical Co., Ltd (Dongguan, China). Deionized water was made at the laboratory. 


\subsection{Synthesis of GTO and EGTO}

The synthesis of the tung oil monoglyceride (GTO) was carried out as described in a previous study [25]. The epoxidized monoglyceride of tung oil (EGTO) was prepared as follows: $100 \mathrm{~g}$ of GTO (0.46 mol carbon-carbon double bond), $\mathrm{CH}_{3} \mathrm{COOH}(0.92 \mathrm{~mol})$ and $\mathrm{TsOH} \cdot \mathrm{H}_{2} \mathrm{O}(0.046 \mathrm{~mol})$ were added to a round-bottom flask. Hydrogen peroxide solution $\left(0.69 \mathrm{~mol} \mathrm{H}_{2} \mathrm{O}_{2}\right)$ was added dropwise over a period of $1 \mathrm{~h}$ and the reaction was allowed to proceed for 4 to $5 \mathrm{~h}$ at a temperature of $55^{\circ} \mathrm{C}$. The EGTO product was neutralized with $1 \mathrm{~mol} / \mathrm{L}$ sodium hydrocarbonate solution and then extracted with ethyl acetate. The ethyl acetate solvent was removed by reduced pressure distillation. EGTO was obtained and its epoxy value was 3\%. The synthesis of GTO and EGTO is illustrated in Scheme 1.

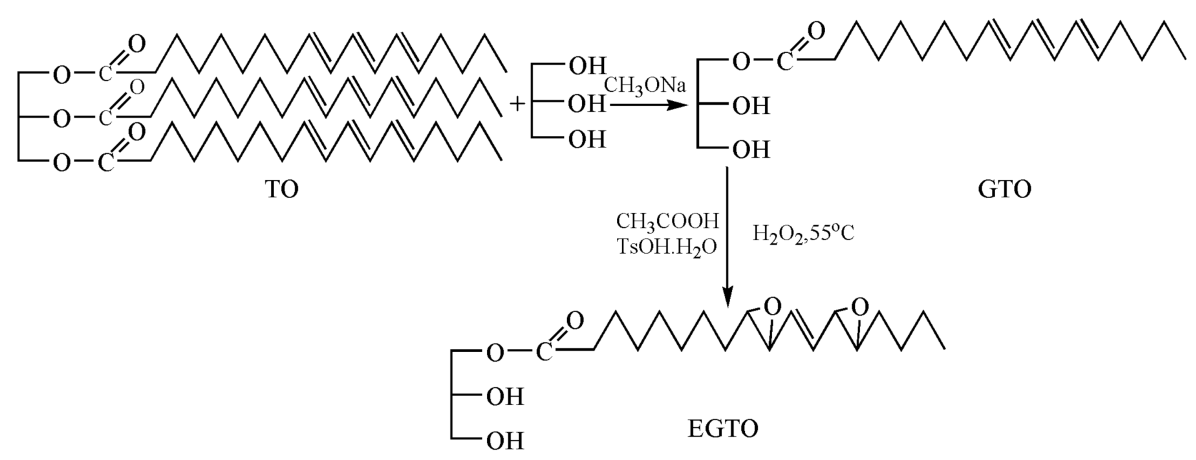

Scheme 1. Synthesis route of tung oil monoglyceride (GTO) and epoxidized monoglyceride of tung oil (EGTO).

\subsection{Synthesis of PTOP and PTOSi}

The reaction equations for PTOP and PTOSi are shown in Schemes 2 and 3 and the parameters for PTOP and PTOSi are summarized in Table 1 . The reaction temperature and time of PTOP were $130{ }^{\circ} \mathrm{C}$ and $120 \mathrm{~min}$, respectively. After the reaction, the product was allowed to cool to room temperature and to stand for $48 \mathrm{~h}$, after which a small amount of unreacted DOPO precipitated as a white solid. The brown clear transparent liquid at the top was washed to neutral with distilled water, which was distilled by vacuum distillation. The conversion rate of DOPO was obtained by comparing the change in the epoxy value of PTOP with that of EGTO. EGTO and DPSD were blended and heated to $140{ }^{\circ} \mathrm{C}$ for $2 \mathrm{~h}$. After the product was cooled down to room temperature and allowed to stand for $48 \mathrm{~h}$, a small amount of the white solid of the unreacted DPSD subsided, the top brown clear transparent liquid was washed to neutral with distilled water and water was removed by reduced pressure distillation. The conversion rate of PTOSi was obtained by comparing the change in the epoxy value of PTOSi with that of EGTO.

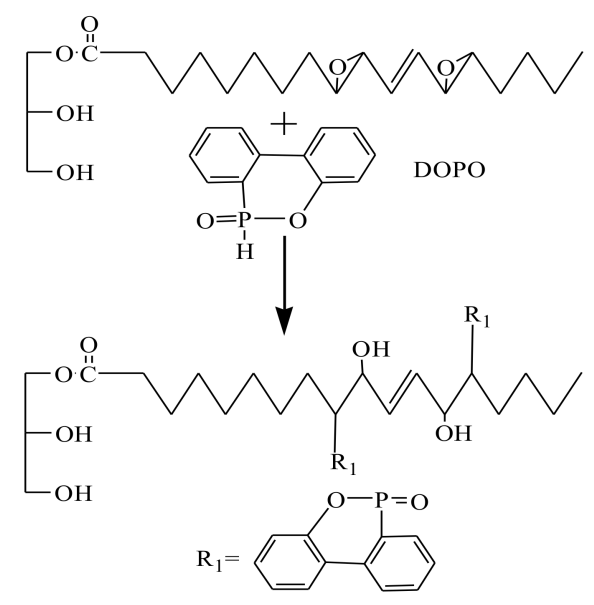

Scheme 2. Synthesis route of phosphorus-containing tung oil-based polyol (PTOP). 


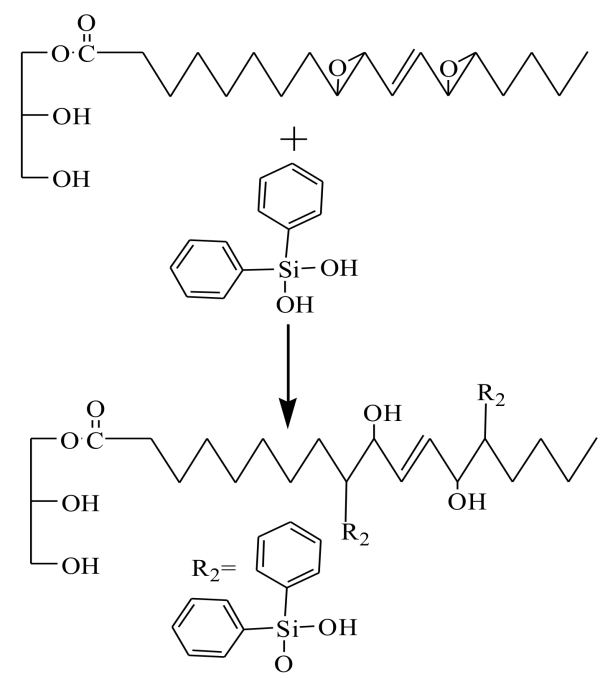

Scheme 3. Synthesis route of silicon-containing tung oil-based polyol (PTOSi).

Table 1. The parameters of PTOP and PTOSi.

\begin{tabular}{ccccccccc}
\hline Sample & $\begin{array}{c}\text { DOPO } \\
(\mathbf{m o l})\end{array}$ & $\begin{array}{c}\text { EGTO/Epoxy } \\
\text { Group }(\mathbf{g} / \mathbf{m o l})\end{array}$ & $\begin{array}{c}\text { DPSD } \\
(\mathbf{m o l})\end{array}$ & $\begin{array}{c}\text { Conversion } \\
\text { Rate } \mathbf{( \% )}\end{array}$ & $\begin{array}{c}\text { Viscosity } \\
\mathbf{( P a} \cdot \mathbf{s})\end{array}$ & $\mathbf{P ~ ( \% )}$ & Si (\%) & $\begin{array}{c}\text { Hydroxyl Value } \\
\mathbf{( m g ~ K O H / g )}\end{array}$ \\
\hline PTOP & 0.072 & $100 / 0.1875$ & 0 & 95.85 & 2.19 & 1.85 & 0 & 339.96 \\
PTOSi & 0 & $100 / 0.1875$ & 0.084 & 95.48 & 2.42 & 0 & 1.95 & 317.93 \\
\hline
\end{tabular}

\subsection{Preparation of RPUF}

RPUF was prepared using a one-step method. The raw materials and formulations used in the preparation of RPUF are listed in Table 2. Firstly, Part A was made up of AK8804, DBTDL, blowing agent, water, PPG4110 and flame-retardant polyols. Then, the mixture of PAPI (Part B) and Part A was stirred for $2 \mathrm{~min}$ at approximately $2000-2500 \mathrm{rpm}$ to ensure homogeneous mixing. At the end, the mixture was poured into a mold and allowed to cure at $80{ }^{\circ} \mathrm{C}$ for $24 \mathrm{~h}$. The P content of blended polyols of RPUF/P60 and RPUF/P80 were $1.11 \%$ and $1.48 \%$, respectively, and the Si content of blended polyols of RPUF/Si60 and RPUF/Si80 were $1.14 \%$ and $1.52 \%$, respectively.

Table 2. Formulation of rigid polyurethane foam (RPUF).

\begin{tabular}{ccccccccc}
\hline \multirow{2}{*}{ Sample } & \multicolumn{7}{c}{ Content Weight (g) } \\
\cline { 2 - 9 } & PPG4110 & PTOP & PTOSi & AK8804 & DBTDL & Water & HFC-365mfc & PAPI \\
\hline Neat RPUF & 100 & 0 & 0 & 2.4 & 1.2 & 1.2 & 20 & 120 \\
RPUF/P60 & 40 & 60 & 0 & 2.4 & 1.2 & 1.2 & 20 & 120 \\
RPUF/P80 & 20 & 80 & 0 & 2.4 & 1.2 & 1.2 & 20 & 120 \\
RPUF/Si60 & 40 & 0 & 60 & 4.0 & 0.28 & 0.17 & 20 & 100 \\
RPUF/Si80 & 20 & 0 & 80 & 4.0 & 0.11 & 0.11 & 20 & 100 \\
\hline
\end{tabular}

\subsection{Characterization}

\subsubsection{Physical Parameter Analysis}

The hydroxyl value and acid value of the sample were determined according to ASTM S957-86 and ASTM D4662-03, respectively. The epoxy value of the sample was tested according to GB/T 1677-2008. The viscosity of the sample was determined based on ASTM D2983 using a rotary Viscometer DVS+ (Brookfield, Middleboro, MA, USA). 


\subsubsection{Gel Permeation Chromatography (GPC)}

The reaction progress of PTOP was tracked by GPC (Waters, Milford, MA, USA). GPC spectra were obtained at a temperature of $30^{\circ} \mathrm{C}$ and flow rate of $1 \mathrm{~mL} / \mathrm{min}$. The parameters of the column were as follows: mixed PL gel $300 \times 718 \mathrm{~mm}, 25 \mu \mathrm{m}$. The solvent was THF.

\subsubsection{Fourier Transform Infrared (FTIR)}

FTIR spectra of each specimen was measured using a Nicolet iS10 FTIR spectrometer (Madison, WI, USA). The wavenumber range was from 500 to $4000 \mathrm{~cm}^{-1}$ at a resolution of $4 \mathrm{~cm}^{-1}$.

\subsubsection{Nuclear Magnetic Resonance (NMR)}

Nuclear magnetic resonance $\left({ }^{1} \mathrm{H}\right.$ NMR) spectra and ${ }^{31}$ PNMR spectra were measured using a BRUKER AV-300 (300MHz) (Bruker BioSpin AG Facilities, Fallanden, Switzerland). Deuterated chloroform was used as the solvent and tetramethylsilane (TMS) and $\mathrm{H}_{3} \mathrm{PO}_{4}$ were used as internal standards.

\subsubsection{Scanning Electron Microscope (SEM)}

The microstructure of the RPUF and RPUF residue was determined by SEM (3400N, Hitachi, Tokyo, Japan).

\subsubsection{Thermogravimetric Analysis (TGA)}

The thermal stability of the specimen was tested using a NETZSCH TG 209F3 (Gebruder-Netzsch-Straße, Germany). Each specimen was heated from 40 to $800{ }^{\circ} \mathrm{C}$ under an $\mathrm{N} 2$ atmosphere with a flow rate of $20 \mathrm{~mL} / \mathrm{min}$ and a heating rate of $10^{\circ} \mathrm{C} / \mathrm{min}$ in alumina crucible.

\subsubsection{Differential Scanning Calorimeter (DSC)}

The thermal property of RPUF was analyzed by DSC on a NETZSCH DSC 200F3 (Gebruder-Netzsch-Straße, Germany). Samples of about $10 \mathrm{mg}$ were weighed and sealed in the aluminum DSC pans and placed in the DSC cell. They were first cooled for $0.5 \mathrm{~min}$ at $-60^{\circ} \mathrm{C}$, and then heated from -60 to $200{ }^{\circ} \mathrm{C}$ at the rate of $20^{\circ} \mathrm{C} / \mathrm{min}$ under nitrogen atmosphere. They were kept at 200 ${ }^{\circ} \mathrm{C}$ for $0.5 \mathrm{~min}$ to eliminate the previous heat history and subsequently cooled to $-60{ }^{\circ} \mathrm{C}$ at $20^{\circ} \mathrm{C} / \mathrm{min}$. Lastly, they were heated again to $200^{\circ} \mathrm{C}$ at $20^{\circ} \mathrm{C} / \mathrm{min}$.

\subsubsection{Limit Oxygen Index (LOI) Test}

The limiting oxygen index test was performed according to ASTM D4986 and using an LOI Meter (PX-01-005, Nanjing Analytical Instrument Factory Co., Ltd, Nanjing, China). The dimensions of the specimen were $100 \times 10 \times 10 \mathrm{~mm}^{3}$. Each sample was run twice and the average result was calculated.

\subsubsection{Cone Calorimeter Test (CTT)}

CTT was carried out by employing a cone calorimeter (FTT2000, Fire Testing Technology, West Sussex, UK) according to ISO5660-1. The dimensions of the sample were $20 \times 100 \times 100 \mathrm{~mm}^{3}$, which was enveloped in aluminum foil. FTT2000 provided the external heat flux at $35 \mathrm{~kW} / \mathrm{m}^{2}$ horizontally. Each specimen was detected two times and the average result was obtained.

\subsubsection{Compressive Strength Test}

The compressive strength of RPUF was measured according to ASTM D1621. It was measured using a CMT4000 universal testing machine (Shenzhen Suns Technology Stock Co., Ltd, Shenzhen, China) where a speed of $2 \mathrm{~mm} / \mathrm{s}$ of compressive force was adopted and each sample was tested 5 times and the average result was obtained. 


\subsubsection{Thermal Conductivity Test}

The thermal conductivity of RPUF was examined using a thermal conductivity analyzer (JB-DZDR-P, Shanghai Jiubin Instrument Co., Ltd, Shanghai, China) according to ASTM C518. The precision of the sample was $50 \times 50 \times 20 \mathrm{~mm}^{3}$.

\subsubsection{Density Test}

The density of RPUF was measured according to ASTM D1622. The measurement of the sample was $50 \times 50 \times 50 \mathrm{~mm}^{3}$, and the density value was obtained from the average of five repeated tests.

\section{Results}

\subsection{Synthesis of PTOP}

The reaction progress of PTOP was monitored by GPC at different times. In Figure 1a, the peak at $17.25 \mathrm{~min}$ was attributed to DOPO, and the broad peak from $10 \mathrm{~min}$ to $15.40 \mathrm{~min}$ was assigned to PTOP, while the narrow peak from $15.40 \mathrm{~min}$ to $16.50 \mathrm{~min}$ was assigned to EGTO. Due to the excessive amount of EGTO, the peak representing EGTO was evident during the PTOP reaction process. The DOPO peak almost disappeared at $2 \mathrm{~h}$, which implies that the reaction between DOPO and EGTO was completed. It can be seen from the Figure 1e,d that no DOPO remained after $2 \mathrm{~h}$ at $130^{\circ} \mathrm{C}$ and $140^{\circ} \mathrm{C}$. High temperatures may cause other side reactions to occur. Thus, the synthesis of PTOP was conducted for $2 \mathrm{~h}$ at a temperature below $130^{\circ} \mathrm{C}$.

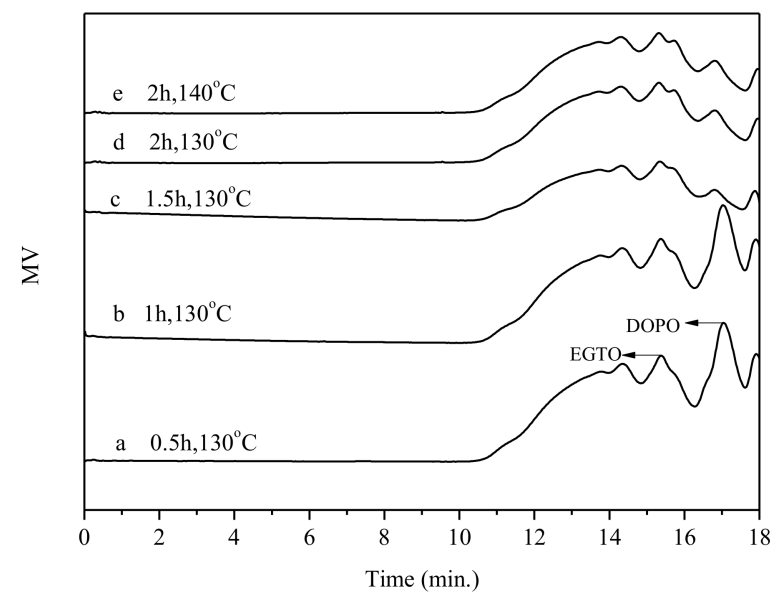

Figure 1. The Gel Permeation Chromatography (GPC) curves of time.

\subsection{FTIR of EGTO, PTOP, and PTOSi}

Figure 2 presents the FTIR spectra of EGTO, PTOP, and PTOSi. Hydroxyl absorption in EGTO appeared at $3440 \mathrm{~cm}^{-1}$, while the $-\mathrm{CH}_{2}$ - stretching vibration peaks appeared at 2930 and $2850 \mathrm{~cm}^{-1}$. The stretching vibration at $900 \mathrm{~cm}^{-1}$ belonged to the epoxy group in EGTO [26]. The characteristic peak for $\mathrm{P}(\mathrm{O})-\mathrm{H}$ stretching of DOPO in PTOP at $2428 \mathrm{~cm}^{-1}$ disappeared, while a broad peak appeared at $3340 \mathrm{~cm}^{-1}$, indicating that the epoxy ring in EGTO opened and a hydroxyl group was formed. Simultaneously, the characteristic-OH peak in PTOP occurred around $3440 \mathrm{~cm}^{-1}$, while the absorption peak at $1590 \mathrm{~cm}^{-1}$ was attributed to $\mathrm{P}-\mathrm{Ph}$, and the absorbing peaks of $\mathrm{P}=\mathrm{O}$ appeared at 1232 and $1198 \mathrm{~cm}^{-1}$. The absorption peaks around 1043 and $918 \mathrm{~cm}^{-1}$ corresponded to $\mathrm{P}-\mathrm{O}-\mathrm{C}$ and $\mathrm{P}-\mathrm{O}-\mathrm{Ph}$ vibrations, which are characteristics of EGTO and DOPO, respectively [27]. The absorption peaks on the FTIR spectrum of PTOSi were consistent with observations from the literature [28]. The presence of a pronounced peak at $1450 \mathrm{~cm}^{-1}$ characteristic of benzene was significant. Peaks at 1070-1130 $\mathrm{cm}^{-1}$ corresponded to $\mathrm{C}-\mathrm{O}$ and $\mathrm{Si}-\mathrm{O}$ bonding [22], and the stretching vibration of two peaks at 880 and 
$730 \mathrm{~cm}^{-1}$ were assigned to the $\mathrm{Si}-\mathrm{C}$ and $\mathrm{C}-\mathrm{H}$ bonds of benzene. All the above changes confirmed that PTOP and PTOSi were formed via the ring opening reaction.

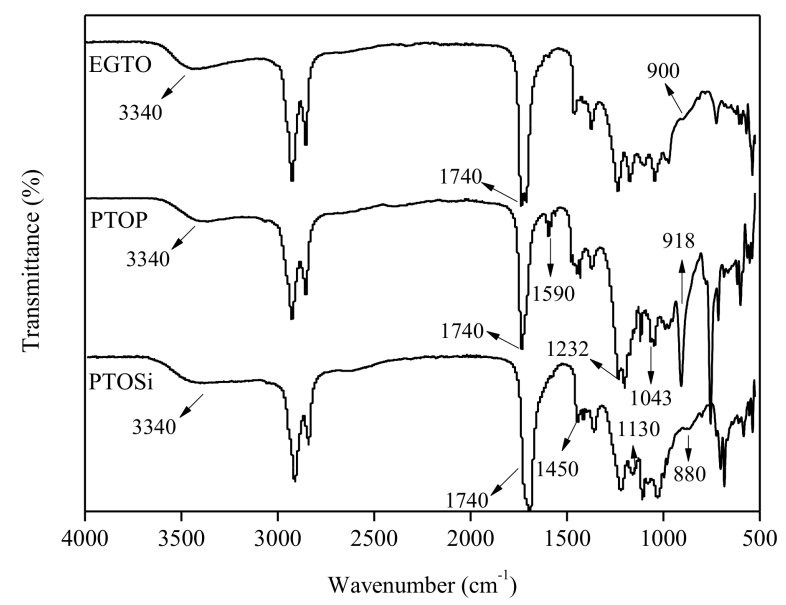

Figure 2. Fourier transfer infrared (FTIR) spectra of EGTO, PTOP, and PTOSi.

\section{3. ${ }^{1} \mathrm{H} N M R$ and ${ }^{31} P$ NMR}

Figure 3a presents peaks at $0.9 \mathrm{ppm}$ and $2.5 \mathrm{ppm}$, which were attributed to methyl protons (peak 1 ) and fatty acid protons [ $\left.\left(-\mathrm{CH}_{2}\right)-\mathrm{CO}-\right]$ (peak 5), respectively. While protons in $-\mathrm{CH}_{2}-$ (peak 6) from glycerol appeared from 4.0 to $4.1 \mathrm{ppm}$, the peak at 5.3 to $5.4 \mathrm{ppm}$ was attributed to the carbon-carbon double bond. The peak at $3.3 \mathrm{ppm}$ in the EGTO spectra indicates that the epoxy group (peak 3) was formed after epoxidation. The peak at $1.9 \mathrm{ppm}$ corresponded to the hydrogen (peak 8) attached to the carbon adjacent to $-\mathrm{OH}$ and the peak that appeared at $2.0 \mathrm{ppm}$ represented the hydrogen atoms adjacent to the ester bond [-O-CO-] (peak 7). In Figure 3b, the chemical shifts at 7.2-8.2 ppm (peak 10) indicated the Ar-H of PTOP, while the absorption peaks at $8.65 \mathrm{ppm}$ (peak 11) and $3.60 \mathrm{ppm}$ (peak 12) represented the $\mathrm{P}-\mathrm{C}-\mathrm{H}$ and $-\mathrm{OH}$ of PTOP, respectively. All of these characteristic ${ }^{1} \mathrm{H}$ NMR bands matched the PTOP structure. In Figure $3 \mathrm{c}$, the peaks at 7.2-8.2 ppm (peak 14) were associated with the Ar-H of PTOSi, while the peak at $3.4 \mathrm{ppm}$ (peak 17) was characteristic of the-OH of DPSD that formed from the epoxy group via a ring opening reaction. The peak at $2.8 \mathrm{ppm}$ (peak 16) was assigned to $\mathrm{Si}-\mathrm{O}-\mathrm{CH}-$, while the $-\mathrm{Si}-\mathrm{OH}$ peak appeared at $2.66 \mathrm{ppm}$ (peak 15). Furthermore, PTOP exhibits a single peak at $35.50 \mathrm{ppm}$ while there is no peak at $14.90 \mathrm{ppm}$ in the ${ }^{31} \mathrm{PNMR}$ spectrum (Figure 4). The chemical shift at $14.90 \mathrm{ppm}$ was attributed to DOPO, which reveals that the reaction between DOPO and EGTO was completed.

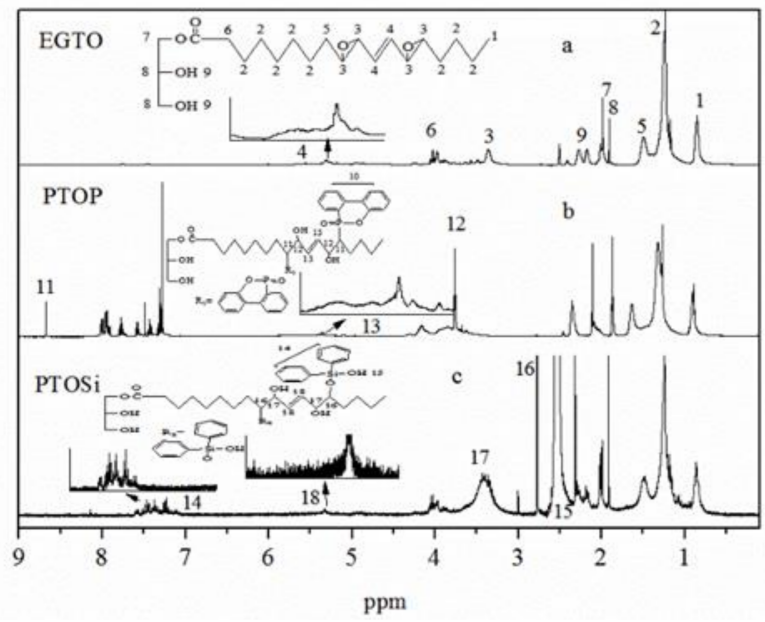

Figure 3. The ${ }^{1} \mathrm{HNMR}$ spectra of EGTO, PTOP, and PTOSi. 


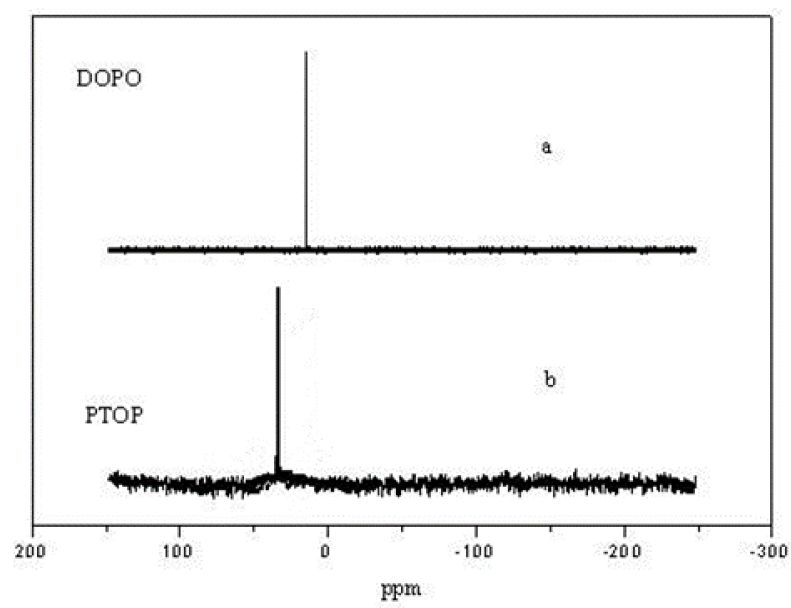

Figure 4. The ${ }^{31} \mathrm{P}$ NMR of DOPO and PTOP.

\subsection{SEM Micrographs of RPUF}

The SEM micrographs of RPUF are presented in Figure 5, where the main structural areas noted are the cells, cell walls, and wall joints [29]. The size range of the cells increased with an increase in PTOP content. This phenomenon was also observed for RPUF/Si60 and RPUF/Si80 with an increase in PTOSi content. It was noted that a number of foam cell orifices and the size of regular cells in RPUF/P60 were consistent with those in RPUF/Si60. However, for RPUF/P80 and RPUF/Si80, the number of cell windows is decreased. The insufficient cross-linking action of PTOP and PTOSi with low functionality leads to a decrease in the number of closed foam cells, and the collapse of the newly formed foam structure due to the decrease in the number of closed foam cells.
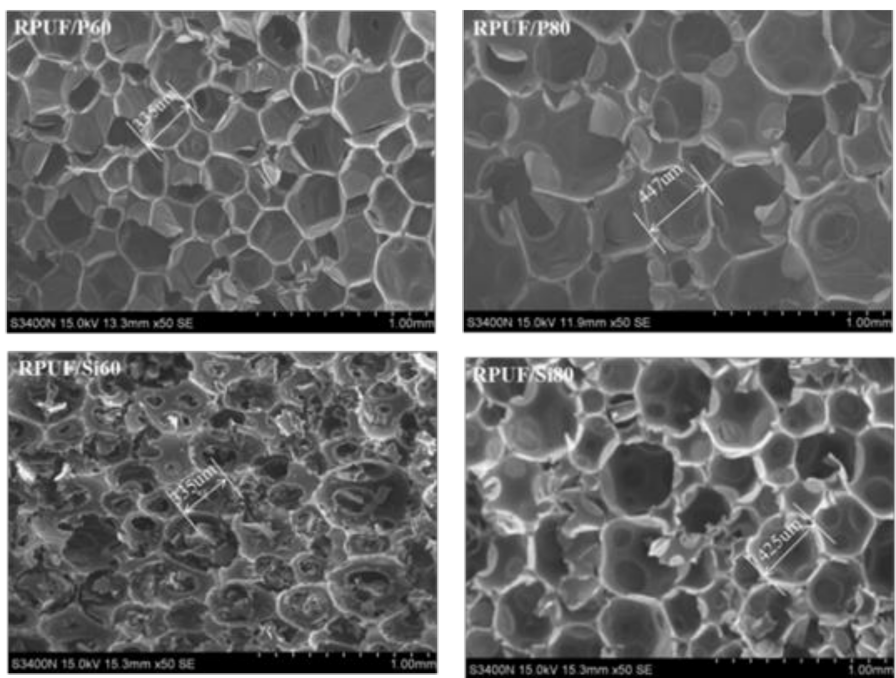

Figure 5. SEM of rigid polyurethane foam (RPUF).

\subsection{Thermal Property of PTOP, PTOSi, and RPUF}

\subsubsection{Thermal stability of PTOP, PTOSi, and RPUF}

Figures 6-8 show the TG and DTG curves of PTOP, PTOSi, and RPUF, respectively. $T_{\text {onset }}$ represents the temperature at $10 \%$ weight loss, $T_{\max }$ represents the temperature when the weight loss rate was maximum, and the residue rate was obtained at $600{ }^{\circ} \mathrm{C}$. These parameters are summarized in Tables 3 and 4. 


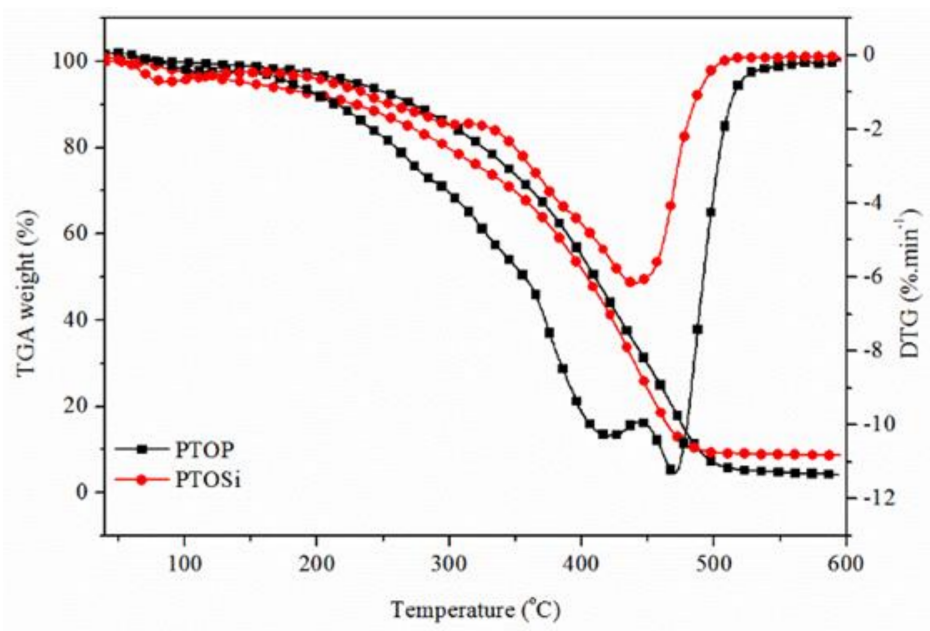

Figure 6. Thermogravimetric (TG) and DTG curves for PTOP and PTOSi.

Table 3. The parameters of TG and DTG of PTOP and PTOSi.

\begin{tabular}{|c|c|c|c|c|}
\hline \multirow{2}{*}{ Sample } & \multicolumn{2}{|c|}{$\mathrm{N}_{2}$ Atmosphere } & \multirow{2}{*}{$\begin{array}{l}\text { The Maximum Decomposition } \\
\text { Rate }(\% / \mathrm{min})\end{array}$} & \multirow{2}{*}{$\begin{array}{l}\text { Residue Rate } \\
\text { (wt \%) }\end{array}$} \\
\hline & $T_{\text {onset }}\left({ }^{\circ} \mathrm{C}\right)$ & $T_{\max }\left({ }^{\circ} \mathrm{C}\right)$ & & \\
\hline PTOP & 272.40 & 468.10 & 11.31 & 4.13 \\
\hline PTOSi & 228.20 & 440.10 & 6.18 & 8.58 \\
\hline
\end{tabular}

As can be seen from Figure 6, PTOP exhibited a higher $T_{\text {onset }}$ than PTOSi, which was attributed to the low temperature of the initial DPSD degradation. Moreover, its $T_{\max }$ was higher than that of PTOSi, while the residue yield of PTOP was less than that of PTOSi. Silicon retained in the chemical bond could be acted as a barrier that prevents the transfer of heat and mass in the condensed phase [30]. These results indicate that the thermal property of PTOP is higher than that of PTOSi.

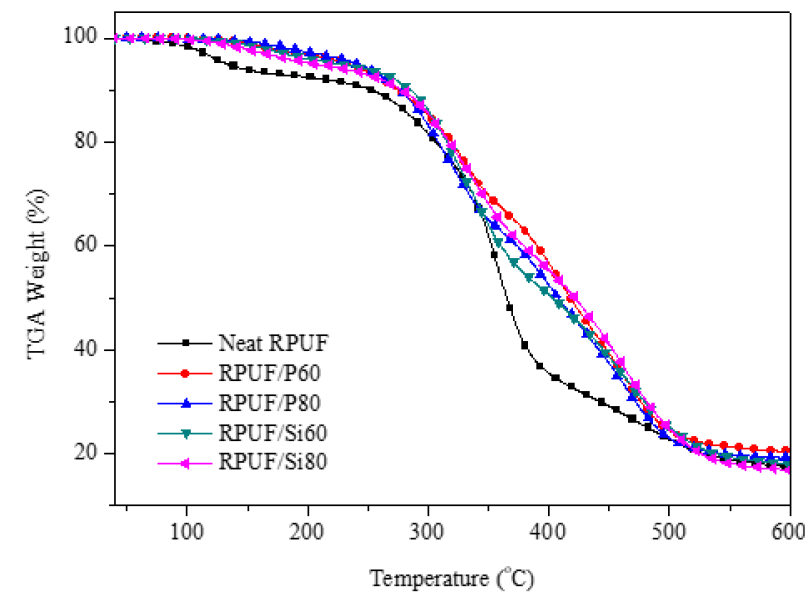

Figure 7. TG curves for RPUF. 


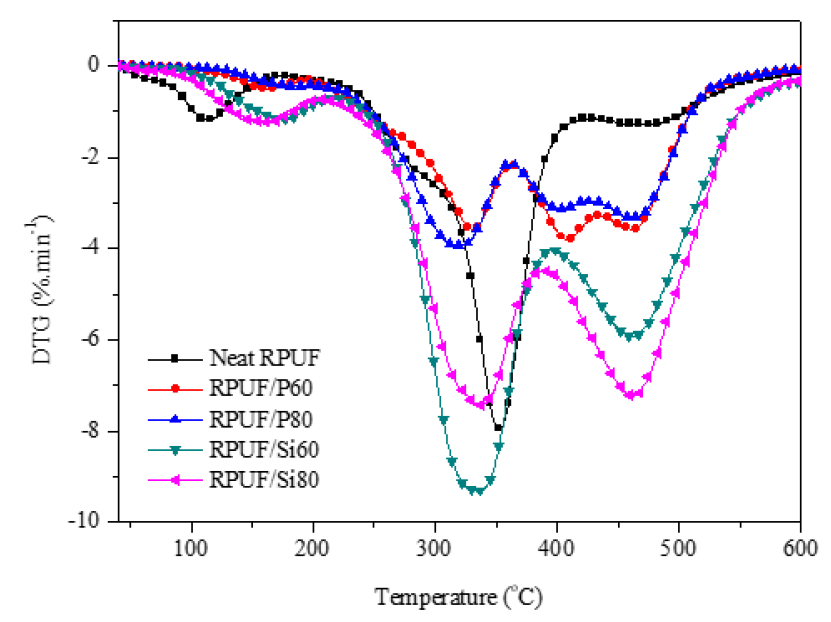

Figure 8. DTG curves for RPUF.

Table 4. The parameters of TG and DTG of RPUF.

\begin{tabular}{cccccc}
\hline \multirow{2}{*}{ Sample } & \multirow{2}{*}{$\boldsymbol{T}_{\text {onset }}\left({ }^{\circ} \mathbf{C}\right)$} & \multicolumn{3}{c}{$\boldsymbol{T}_{\max }\left({ }^{\circ} \mathbf{C}\right)$} & \multirow{2}{*}{ Residue Rate (wt \%) } \\
\cline { 3 - 5 } & & Step I & Step II & Step III & \\
\hline Neat RPUF & 313.10 & 354.30 & 478.10 & $/$ & 17.54 \\
RPUF/P60 & 282.60 & 330.90 & 408.70 & 461.40 & 20.48 \\
RPUF/P80 & 261.80 & 319.60 & 402.30 & 460.60 & 19.20 \\
RPUF/Si60 & 291.40 & 335.10 & 463.70 & $/$ & 18.03 \\
RPUF/Si80 & 288.50 & 338.60 & 464.50 & $/$ & 17.00 \\
\hline
\end{tabular}

Thermal degradation of RPUF/P60 and RPUF/P80 showed four stages at 100 to $200{ }^{\circ} \mathrm{C}, 200$ to 350 ${ }^{\circ} \mathrm{C}, 350$ to $450{ }^{\circ} \mathrm{C}$, and 450 to $600{ }^{\circ} \mathrm{C}$, respectively. The first stage was attributed to the entrapment of volatile compounds in closed cells, while the other stages were attributed to the hard and soft segments of polyurethane. With an increase in the PTOP content of RPUF, the residue rate at $600{ }^{\circ} \mathrm{C}$ after complete decomposition becomes smaller, indicating that the degradation products of DOPO have mainly decomposed to form volatile products. The initial decomposition temperature of RPUF/P80 was lower than that of RPUF/P60, and the decreased $T_{\text {onset }}$ could be attributed to the prior chemical decomposition of DOPO compared to PPG4110. Step I of $T_{\max }$ was associated with the urethane unit, which was considered as the hard segment in the polyurethane network. Step II of $T_{\max }$ was attributed to the soft segments, which were mainly made up of long chains of PPG4110 and PTOP. While step III of $T_{\max }$ was affected by the aromatic components in RPUF, the aromatic components were released within the temperature range of 400 to $600^{\circ} \mathrm{C}$ [31]. The condensation, chain scission, and cross-linking reactions of PTOP and the decomposition mechanism of PTOP in RPUF were summarized in that acid source, and polyphosphate compound could be produced via degradation in the step III.

Two stages showed in the degradation process of neat RPUF, RPUF/Si60, and RPUF/Si80. The initial thermal temperature and $T_{\max }$ of neat RPUF were highest among the five samples, due to the hydroxyl functionality of PPG4110 was higher than those for PTOP and PTOSi, the relative molecular weight and the cross-linking yield of neat RPUF were highest. While the maximum decomposition rate was higher than these for other samples, results reveal that the neat RPUF was degraded quickly when the temperature was higher than the initial thermal temperature.

The initial thermal decomposition and residue rates of RPUF/Si60 and RPUF/Si80 were similar to those for RPUF/P60 and RPUF/P80, while the change in following characteristic parameters such as $T_{\max }$ were contrary. The degradation of RPUF/Si60 and RPUF/Si80 was divided into two steps. The original degradation was ascribed to the urethane unit in the cross-linking network. Substances containing benzene structures and Si-containing groups in the RPUF were degraded when the temperature was raised. 


\subsubsection{DSC of RPUF}

Thermal property of RPUF was also studied by DSC, the corresponding heating thermograms are shown in Figure 9. The molecular chain of polyurethane foam was generally composed of the soft segment and hard segment. At the room temperature, soft segment was in an elastomeric state, which has an effect on low temperature performance, flexibility, organic solvent resistance and the weather-ability on RPUF. While the hard segment was in a glassy or crystalline state, which affects the melting point, thermal stability, hardness and modulus of RPUF. The temperature range of glassy transition of RPUF was from 29 to $102^{\circ} \mathrm{C}$, the $T_{\mathrm{g}}$ of RPUF/P60, RPUF/P80, RPUF/Si60, and RPUF/Si80 were $70.72,65.08,73.17$, and $77.95^{\circ} \mathrm{C}$, respectively. The soft segment was made up of the tung oil-based flame retardant polyols and PPG4110, the hard segment was made up of isocyanate. The reason why the $T_{\mathrm{g}}$ of RPUF was less when increasing the amount of PTOP was that PTOP could be acted as the soft segment. On the contrary, with increasing the amount of PTOSi, the $T_{\mathrm{g}}$ of RPUF was higher due to PTOSi could be acted as soft segment.

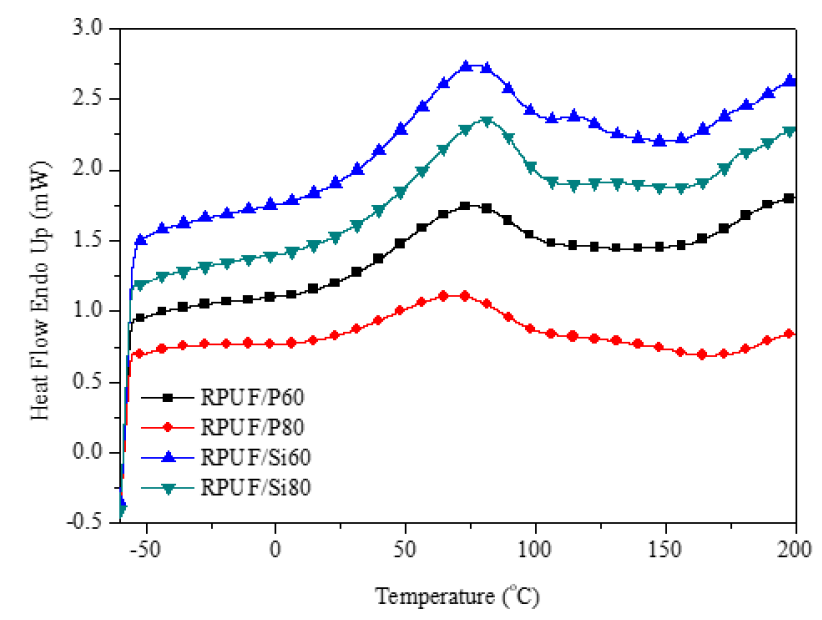

Figure 9. Differential Scanning Calorimetry (DSC) of RPUF.

\subsection{Flame Retardancy}

\subsubsection{Limiting Oxygen Index (LOI) Test of RPUF}

Air usually contains almost $21 \% \mathrm{O}_{2}$, so materials with an LOI value of $21 \%$ or less will burn quickly in air [6]. With the accession of PTOP and PTOSi, the LOI value of RPUF increased, indicating that both of these are flame retardant polyols. The LOI values for RPUF are listed in Table 5, where it can be seen that they are higher than that of neat RPUF, which is $19.0 \%$. With increasing amounts of PTOP and PTOSi, the LOI value increased from $21.3 \%$ for RPUF/P60 to $24.0 \%$ for RPUF/P80, while a lower increase in the LOI value was observed for RPUF/Si60 and RPUF/Si80 from $21.0 \%$ to $23.4 \%$. This result was attributed to the large number of $\mathrm{PO}^{-}, \mathrm{PO}_{2}{ }^{-}$, and $\mathrm{P}_{2} \mathrm{O}_{4}{ }^{-}$. Free radicals that were generated and eliminated the combustible gas during the gaseous phase. Meanwhile, phosphate and its derivatives were formed from $\mathrm{PO}^{-}, \mathrm{PO}_{2}{ }^{-}$, and $\mathrm{P}_{2} \mathrm{O}_{4}{ }^{-}$, and served as a barrier to control the transfer of heat and combustible gas during the burning process [24]. During the combustion of RPUF/Si60 and RPUF/Si80, silicones can effectively shield via silica residue left when under oxygen at elevated temperatures [32]. Silica plays the role of an "insulating blanket" as a barrier to delay volatile mass transport during the decomposition. In Table 5, the LOI value of RPUF. Based on the LOI value of RPUF, PTOP showed better flame-retardant performance than that of PTOSi. 
Table 5. RPUF Limiting Oxygen Index (LOI) values.

\begin{tabular}{cccccc}
\hline Sample & Neat RPUF & RPUF/P60 & RPUF/P80 & RPUF/Si60 & RPUF/Si80 \\
\hline LOI value $(\%)$ & 19.0 & 21.3 & 24.0 & 21.0 & 23.4 \\
\hline
\end{tabular}

\subsubsection{Fire Behaviors of RPUF}

The heat release rate (HRR), peak of heat release rate (PHRR), time to ignition (TII), and total heat released (THR) measured for RPUF during the cone calorimetry test are presented in Table 6.

Table 6. Cone calorimeter testing data for RPUF.

\begin{tabular}{|c|c|c|c|c|}
\hline Sample & TII (s) & PHRR $\left(\mathrm{kW} \cdot \mathrm{m}^{-2}\right)$ & Time to PHRR (s) & THR $\left(\mathrm{MJ} \cdot \mathrm{m}^{-2}\right)$ \\
\hline RPUF/P60 & 2 & 266.03 & 35,75 & 22.78 \\
\hline RPUF/P80 & 3 & 259.38 & 40,60 & 18.40 \\
\hline RPUF/Si60 & 2 & 285.36 & 30,60 & 22.80 \\
\hline RPUF/Si80 & 3 & 255.20 & 40,65 & 21.99 \\
\hline
\end{tabular}

Very similar HRR curves are obtained for RPUF in Figure 10 when the PTOP and PTOSi content is increased, where the curves are seen to decrease but then quickly increase to attain the second HRR peak. RPUF ignited within the first few seconds (about 2-3 s) of exposure to heat burned steadily for 175 to $230 \mathrm{~s}$. The first sharp peaks for RPUF were turned up at 35, 40, 30, and 40 s, respectively. Then, narrow peaks for each RPUF appeared at 75, 60, 60, and $65 \mathrm{~s}$. The protective char layer formed from the first peak in the HRR curves, then the protective char layer was degraded, which is represented by the second peak was [33]. The HRR peaks for RPUF/P80 and RPUF/Si80 were slightly smaller and thinner, while the peak times were lengthened with an increase in the amount of PTOP and PTOSi. This may be attributed to the introduction of PTOP and PTOSi into the soft segments, contributing to the formation of the protective and continuous char layer.

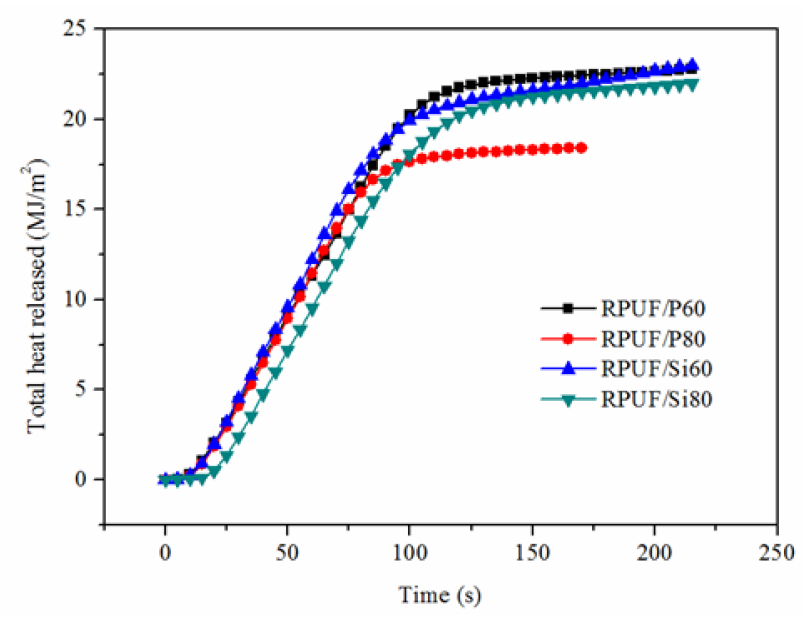

Figure 10. Heat release rate (HRR) curves of RPUF.

THR curves in Figure 11 illustrate that the value of THR decreases in the order of RPUF/P60>RPUF/Si60>RPUF/Si80>RPUF/P80. For example, the THR of RPUF/P80 is $18.40 \mathrm{MJ} \cdot \mathrm{m}^{-2}$, a $19.30 \%$ reduction compared to that of RPUF/P60. There are two reasons for this result. On the one hand, heat is taken away by the condensed-phase and gas-phase actions of the volatile aromatic phosphates in RPUF/P60 and RPUF/P80. On the other hand, the barrier layer produced by PTOP and PTOSi can prevent heat, oxygen and flammable substance from transferring. Moreover, non-flammable gas in the gas phase system can also effectively reduce the heat released from RPUF/P80 [34]. In comparing to RPUF/Si60, RPUF/Si80 has the preferable flame-retardant property indicated by 
both the LOI value and CTT. The excellent flame-retardant behavior of PTOSi was illustrated by its better dispersion within the RPUF and movement towards the RPUF surface during the burning process. A highly flame-retardant char generated from the combustion of PTOSi can reduce the total heat released.

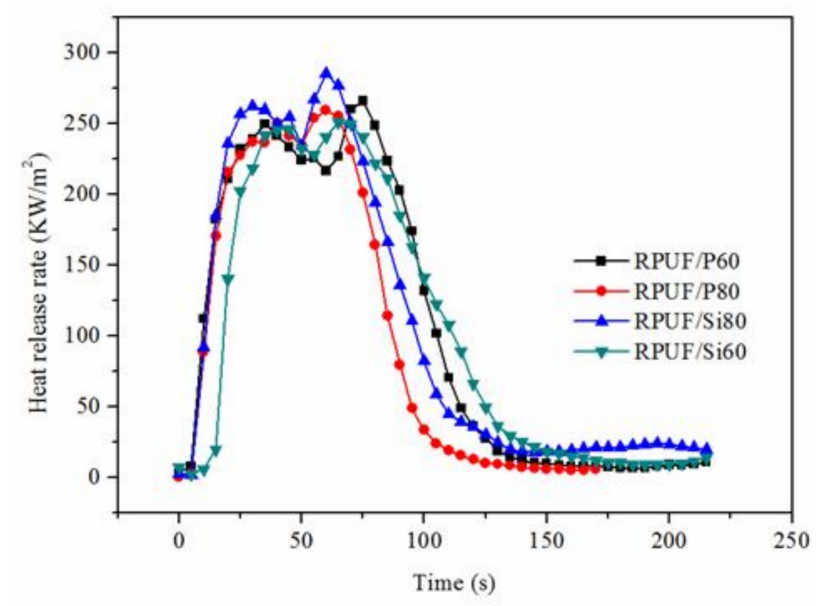

Figure 11. Total heat released (THR) curves of RPUF.

Based on related parameters such as PHRR and THR presented in Table 6, the flame-retardant efficiency of PTOP is higher than that of PTOSi. This result accounts for the fact that PTOP can migrate to the surface of the RPUF during combustion and allow for the formation of the condensed-phase and gas-phase of volatile aromatic phosphates. This indicates that the addition of PTOP and PTOSi can boost the fire-inhibition activity during combustion.

\subsubsection{SEM and EDAX of RPUF Residue}

To confirm that P-containing compounds and Si-containing compounds were retained in the residue and acted as the flame retardant barrier. The microstructure of the residue after combustion was examined by SEM, RPUF/P60, RPUF/P80, RPUF/Si60, and RPUF/Si80 had the compacted and smooth char. EDAX (energy dispersive spectroscopy) associated with SEM was used to get the elemental composition of residues of RPUF. Table 7 shows the surface composition of the residues obtained by EDAX. As can be observed from Table 7, $\mathrm{C}$ and $\mathrm{O}$ were the main remaining elements. The char layer of RPUF/P60 and RPUF/P80 can compress well to form a protective phosphatic barrier to resist the transfer of heat and mass during fire. As shown in Figure 12, a number of flaws were found on the surface of RPUF/Si60 and RPUF/Si80, which may be the result of $\mathrm{SiO}_{2}$ forming and moving onto the surface during burning. PTOP and PTOSi have the effect of promoting the char layers, as compacted char layers may prevent the internal products of pyrolysis from transferring to the flame zone.

Table 7. EDAX data of the residue of RPUF.

\begin{tabular}{|c|c|c|c|c|c|}
\hline Sample & $C(w t \%)$ & $\mathrm{O}(w \mathrm{t} \%)$ & $P(w t \%)$ & Si (wt \%) & Others (wt \%) \\
\hline RPUF/P60 & 79.67 & 17.72 & 1.83 & 0 & 0.78 \\
\hline RPUF/P80 & 83.38 & 14.35 & 0.83 & 0 & 0.84 \\
\hline RPUF/Si60 & 77.37 & 20.61 & 0 & 1.13 & 0.89 \\
\hline RPUF/Si80 & 79.61 & 17.08 & 0 & 2.31 & 1.01 \\
\hline
\end{tabular}



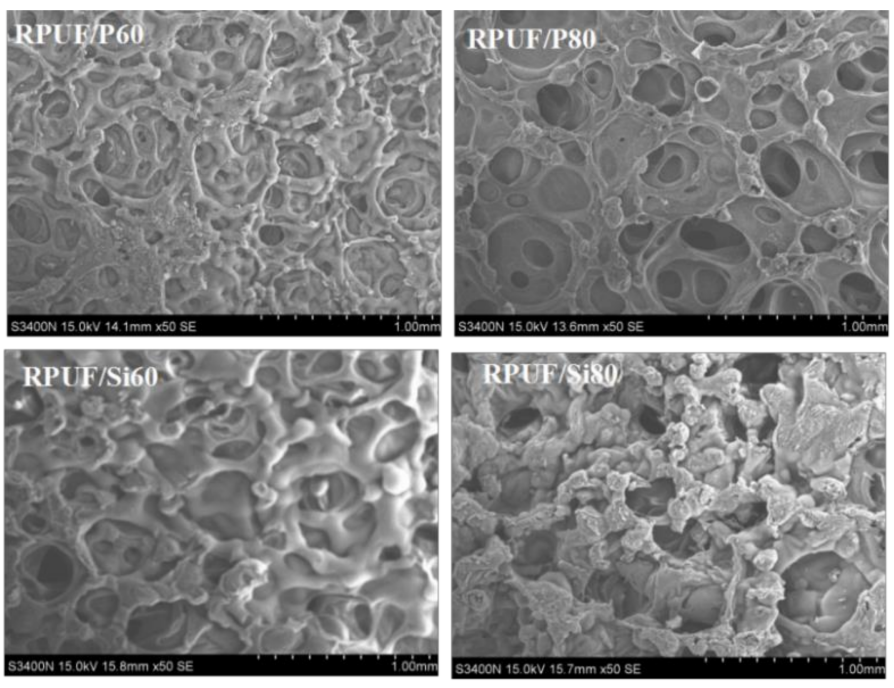

Figure 12. SEM of RPUF residue.

\subsection{Mechanical Property of RPUF}

Mechanical property is an essential issue for the practical application of RPUF, which was further investigated, such as density, compressive strength and thermal conductivity. In order to eliminate the effect of RPUF density on the compressive strength, the specific compressive strength (compressive strength/density) was used to for comparison.

Table 8 shows that the trend in RPUF density is opposite to the trend noted for compressive strength. With large amounts of PTOP and PTOSi added into the blended polyols component, the PTOP and PTOSi may disperse poorly, which can interfere with the development of the cell and make the cell size larger and the number of closed cells lower (Figure 5). PTOP can reduce the compressive strength of RPUF, while the higher content of PTOSi incorporated into RPUF can increase the compressive strength. A similar result was obtained for $\sigma_{\text {sp. }}$. When the PTOP content was below $60 \%$, PTOP dispersed well in the blended polyols component, and almost no agglomeration appeared. PTOSi with its self-crosslinking function can make the RPUF matrix easily collapsible due to the wall fragility of the foam cell, resulting in the worse value of compressive strength.

Table 8. Physical and mechanical properties of RPUF.

\begin{tabular}{ccccc}
\hline Sample & $\boldsymbol{\rho}\left(\mathbf{k g} \cdot \mathbf{m}^{-\mathbf{3}}\right)$ & $\boldsymbol{\sigma} \mathbf{( M P a})$ & $\boldsymbol{\sigma}_{\text {sp }} \mathbf{( N . m . \mathbf { k g } ^ { - \mathbf { 1 } } )}$ & $\mathbf{K}\left(\mathbf{W} \cdot \mathbf{m k} \mathbf{k}^{\mathbf{1}} \mathbf{)}\right.$ \\
\hline RPUF/P60 & $97.08 \pm 0.44$ & $0.82 \pm 0.02$ & 8446 & 0.039 \\
RPUF/P80 & $89.48 \pm 0.80$ & $0.75 \pm 0.03$ & 8381 & 0.038 \\
RPUF/Si60 & $57.09 \pm 0.38$ & $0.18 \pm 0.02$ & 3152 & 0.051 \\
RPUF/Si80 & $52.94 \pm 0.32$ & $0.25 \pm 0.02$ & 4722 & 0.044 \\
\hline
\end{tabular}

$\rho$ : density; $\sigma$ : compressive strength; $\sigma_{\mathrm{sp}}$ : specific compressive strength; $\mathrm{K}$ : thermal conductivity.

RPUF is also used as the thermal insulation material in building insulation. The best method for improving the sustainability of building insulation is to diminish the thermal conductivity of RPUF. With an increase in the PTOP and PTOSi content, the thermal conductivity of RPUF decreased as the thermal conductivity is mainly affected by the average cell size. The thermal conductivity of RPUF/Si60 and RPUF/Si80 were higher than that of RPUF/P60 and RPUF/P80 due to the lower closed cell content, which is influenced by the degree of cross-linking, the equivalent weight of blended polyols and the content of various agents. Therefore, the molecular weight of polyurethane in RPUF/P60 and RPUF/P80 was lower than that for RPUF/Si60 and RPUF/Si80. 


\section{Conclusions}

A novel method was developed to synthesize PTOP and PTOSi, which were proved to be effective flame-retardant polyols used in the formation of RPUF with excellent flame retardancy. Different PTOP and PTOSi contents affect the thermal stability, flame retardancy, and mechanical properties of RPUF. Results from FTIR, TG, DSC, and LOI revealed that DOPO and DPSD are linked to EGTO by a covalent bond, and the thermal stability of PTOP was higher than that for PTOSi. The thermal stability and flame retardancy of RPUF increased, with the LOI value of RPUFs with $80 \%$ PTOP and PTOSi reaching $24.0 \%$ and $23.4 \%$, respectively. PTOP and PTOSi could be acted as the soft segment and hard segment in RPUF, respectively. The compressive strength of RPUF was reduced to $0.75 \mathrm{MPa}$ with $80 \mathrm{wt} \% \mathrm{PTOP}$, while the compressive strength of RPUF increased to $0.25 \mathrm{MPa}$ with $80 \mathrm{wt} \%$ PTOSi. A comparison of the flame-retardant mechanism of RPUF prepared by PTOP and PTOSi showed that RPUF with PTOP released an acid source and polyphosphate compound while RPUF with PTOSi burned out silicon dioxide on the surface. Based on the LOI value of RPUF, PTOP showed better flame-retardant performance than that of PTOSi.

Author Contributions: W.Z. and M.Z. conceived and designed the experiments; C.B., P.J., and Y.Z. performed the experiments and analyzed the data; W.Z. wrote the paper.

Funding: This research was funded by the National Natural Science Foundation of China grant number [31670577 and 31670578].

Conflicts of Interest: The authors declare no conflict of interest.

\section{References}

1. Sylwia, C.; Massimo, F.B.; Jan, K.; Anna, S.; Marcin, M.; Krzysztof, S. Linseed oil as a natural modifier of rigid polyurethane foams. Ind. Crops Prod. 2018, 115, 40-51.

2. Lligadas, G.; Ronda, J.C.; Galia, M.; Cadiz, V. Renewable polymeric materials from vegetable oils: A perspective. Mater. Today 2013, 16, 337-343. [CrossRef]

3. Athanasia, A.S.; David, A.C.E.; Celine, C.; Darren, J.M.; Pratheep, K.A. A systematic study substituting polyether polyol with palm kernel oil based polyester polyol in rigid polyurethane foam. Ind. Crops Prod. 2015, 66, 16-26.

4. Zhou, X.; Sain, M.M.; Oksman, K. Semi-rigid biopolyurethane foams based on palm-oil polyol and reinforced with cellulose nanocrystals. Compos. Part A Appl. Sci. Manuf. 2015, 83, 56-62. [CrossRef]

5. Zieleniewska, M.; Leszczynski, M.K.; Kuranska, M.; Prociak, A.; Szczepkowski, L.; Krzyzowska, M.; Ryszkowska, J. Preparation and characterisation of rigid polyurethane foams using a rapeseed oil-based polyol. Ind. Crops Prod. 2015, 74, 887-897. [CrossRef]

6. Shirke, A.G.; Dholakiya, B.Z.; Kuperkar, K. Modification of tung oil-based polyurethane foam by anhydrides and inorganic content through esterification process. J. Appl. Polym. Sci. 2017, 135, 45786. [CrossRef]

7. Wang, C.; Wu, Y.C.; Li, Y.C.; Shao, Q.; Yan, X.G.; Han, C.; Wang, Z.; Liu, Z.; Guo, Z.H. Flame-retardant rigid polyurethane foam with a phosphorus-nitrogen single intumescent flame retardant. Polym. Adv. Technol. 2018, 29, 668-676. [CrossRef]

8. Shi, X.X.; Jiang, S.H.; Zhu, J.Y.; Li, G.H.; Peng, X.F. Establishment of a highly efficient flame-retardant system for rigid polyurethane foams based on biphase flame-retardant actions. RSC Adv. 2018, 8, 9985-9995. [CrossRef]

9. Shi, S.Y.; Neisius, M.; Mispreuve, H.; Naescher, R.; Gaan, S. Flame retardancy and thermal decomposition of flexible polyurethane foams: Structural influence of organophosphorus compounds. Polym. Degrad. Stab. 2012, 97, 2428-2440.

10. Thirumal, M.; Khastgir, D.; Nando, G.B.; Naik, Y.P.; Singha, N.K. Halogen-free flame retardant PUF: Effect of melamine compounds on mechanical, thermal and flame retardant properties. Polym. Degrad. Stab. 2010, 95, 1138-1145. [CrossRef]

11. Chattopadhyay, D.K.; Webster, D.C. Thermal stability and flame retardancy of polyurethanes. Prog. Polym. Sci. 2009, 34, 1068-1133. [CrossRef] 
12. Laoutid, F.; Bonnaud, L.; Alexandre, M.; Lopez-Cuesta, J.M.; Dubosi, P. New prospects in flame retardant polymer materials: From fundamentals to nanocomposites. Mater. Sci. Eng. R Rep. 2009, 63, $100-125$. [CrossRef]

13. Perret, B.; Schartel, B.; Stob, K.; Ciesielski, M.; Diederichs, J.; Doring, M.; Kramer, J.; Altstadt, V. Novel DOPO-based flame retardants in high-performance carbon fibre epoxy composites for aviation. Eur. Polym. 2011, 47, 1081-1089. [CrossRef]

14. Li, J.L.; Li, Z.W.; Wang, H.G.; Wu, Z.J.; Wang, Z.; Li, S.C. Liquid oxygen compatibility and cryogenic mechanical properties of a novel phosphorous/silicon containing epoxy-based hybrid. RSC Adv. 2016, 6, 91012-91023. [CrossRef]

15. Gaan, S.; Liang, S.Y.; Mispreuve, H.; Perler, H.; Naescher, R. Flame retardant flexible polyurethane foams from novel DOPO-phosphonamidate additives. Polym. Degrad. Stab. 2015, 113, 180-188. [CrossRef]

16. Granzow, A. Flame retardation by phosphorus compounds. Acc. Chem. Res. 1978, 11, 177-183. [CrossRef]

17. Zhang, M.; Luo, Z.Y.; Zhang, J.W.; Chen, S.G.; Zhou, Y.H. Effects of a novel phosphorus-nitrogen flame retardant on rosin-based rigid polyurethane foams. Polym. Degrad. Stab. 2015, 120, 427-434. [CrossRef]

18. Liu, Y.L.; He, J.Y.; Yang, R.J. The thermal properties and flame retardancy of 9, 10-dihydro-9-oxa-10-phosphaphenanthrene-10-oxide (10)-Mg/Polyisocyanate -polyurethane foam composites. Bull. Chem. Soc. Jpn. 2016, 89, 779-785. [CrossRef]

19. Shi, Y.C.; Wang, G.J. The novel silicon-containing epoxy/PEPA phosphate flame retardant for transparent intumescent fire resistant coating. Appl. Surf. Sci. 2016, 385, 453-463. [CrossRef]

20. Wang, J.H.; Ji, C.T.; Yan, Y.T.; Zhao, D.; Shi, L.Y. Mechanical and ceramifiable properties of silicone rubber filled with different inorganic fillers. Polym. Degrad. Stab. 2015, 121, 149-156. [CrossRef]

21. Bian, X.C.; Tang, J.H.; Li, Z.M. Flame retardancy of whisker silicon oxide/rigid polyurethane foam composites with expandable graphite. J. Appl. Polym. Sci. 2010, 110, 3871-3879. [CrossRef]

22. Zhou, H.F.; Wang, H.; Tian, X.Y.; Zheng, K.; Cheng, Q.T. Effect of 3-Aminopropyltriethoxysilane on polycarbonate based waterborne polyurethane transparent coatings. Prog. Org. Coat. 2014, 77, 1073-1078. [CrossRef]

23. Zhang, L.; Wang, Y.C.; Liu, Q.; Cai, X.F. Synergistic effects between silicon-containing flame retardant and DOPO on flame retardancy of epoxy resins. J. Therm. Anal. Calorim. 2016, 123, 1343-1350. [CrossRef]

24. Li, M.L.; Zhong, Y.; Wang, Z.; Fischer, A.; Ranft, F.; Drummer, D.; Wu, W. Flame retarding mechanism of polyamide 6 with phosphorus-nitrogen flame retardant and DOPO derivatives. J. Appl. Polym. Sci. 2016, 133. [CrossRef]

25. Zhou, W.; Jia, P.Y.; Zhou, Y.H.; Zhang, M. Preparation and characterization of tung oil-based flame retardant polyols. Chin. J. Chem. Eng. 2018. [CrossRef]

26. Zhang, Y.C.; Xu, G.L.; Liang, Y.; Yang, J.; Hu, J. Preparation of flame retardant epoxy resins containing DOPO group. Thermochim. Acta 2016, 643, 33-40. [CrossRef]

27. Xiong, Y.Q.; Zhang, X.Y.; Liu, J.; Li, M.M.; Guo, F.; Xia, X.N.; Xu, W.J. Synthesis of novel phosphorus-containing epoxy hardeners and thermal stability and flame-retardant properties of cured products. J. Appl. Polym. Sci. 2012, 125, 1219-1225. [CrossRef]

28. Han, M.S.; Kim, Y.H.; Han, S.J.; Choi, S.J.; Kim, S.B.; Kim, W.N. Effects of a silane coupling agent on the exfoliation of organoclay layers in polyurethane/organoclay nanocomposite foams. J. Appl. Polym. Sci. 2008, 110, 376-386. [CrossRef]

29. Chen, M.J.; Chen, C.R.; Tan, Y.; Huang, J.Q.; Wang, X.L.; Chen, L. Inherently flame-retardant flexible polyurethane foam with low content of phosphorus-containing cross-linking agent. Ind. Eng. Chem. Res. 2014, 53, 1160-1171. [CrossRef]

30. Wu, K.; Song, L.; Hu, Y.; Lu, H.D.; Kandola, B.K.; Kandare, E. Synthesis and characterization of a functional polyhedral oligomeric silsesquioxane and its flame retardancy in epoxy resin. Prog. Org. Coat. 2009, 65, 490-497. [CrossRef]

31. Zhang, W.C.; Li, X.M.; Yang, R.J. Pyrolysis and fire behavior of epoxy resin composites based on a phosphorus-containing polyhedral oligomeric silsesquioxane (DOPO-POSS). Polym. Degrad. Stab. 2011, 96, 1821-1832. [CrossRef]

32. Hamdani, S.; Longuet, C.; Perrin, D.; Lopez-cuesta, J.M.; Ganachaud, F. Flame retardancy of silicone-based materials. Polym. Degrad. Stab. 2009, 94, 465-495. [CrossRef] 
33. Wu, X.; Wang, L.; Wu, C.; Yu, J.; Xie, L.; Wang, G.; Jiang, P. Influence of char residues on flammability of EVA/EG, EVA/NG and EVA/GO composites. Polym. Degrad. Stab. 2012, 97, 54-63. [CrossRef]

34. Zhao, B.; Chen, L.; Long, J.W.; Chen, H.B.; Wang, Y.Z. Aluminum hypophosphite versus alkyl-substituted phosphinate in polyamide 6: Flame retardance, thermal degradation, and pyrolysis behavior. Ind. Eng. Chem. Res. 2013, 52, 2875-2886. [CrossRef] 